\title{
Philosophiques
}

\section{Lucie Antoniol, Lire Ryle aujourd'hui. Aux sources de la philosophie analytique. De Boeck, Bruxelles, 1993, 133 p.}

\section{Mathieu Marion}

Volume 22, numéro 2, automne 1995

URI : https://id.erudit.org/iderudit/027353ar

DOI : https://doi.org/10.7202/027353ar

Aller au sommaire du numéro

Éditeur(s)

Société de philosophie du Québec

ISSN

0316-2923 (imprimé)

1492-1391 (numérique)

Découvrir la revue

Citer ce compte rendu

Marion, M. (1995). Compte rendu de [Lucie Antoniol, Lire Ryle aujourd'hui. Aux sources de la philosophie analytique. De Boeck, Bruxelles, 1993, 133 p.]

Philosophiques, 22(2), 518-522. https://doi.org/10.7202/027353ar d'utilisation que vous pouvez consulter en ligne.

https://apropos.erudit.org/fr/usagers/politique-dutilisation/ 


\section{Lucie Antoniol, Lire Ryle aujourd'hui. Aux sources de la philosophie analytique. De Boeck, Bruxelles, 1993, $133 \mathrm{p}$.}

\section{par Mathieu Marion}

Gilbert Ryle fut une des figures dominantes du monde philosophique oxonien de l'après guerre, dont il a contribué à façonner certains de ses traits les plus distinctifs. Dans sa courte étude Lire Ryle aujourd'hui, la première en langue française sur le sujet, Lucie Antoniol présente une interprétation somme toute assez juste (à défaut d'être originale) des grands thèmes de l'ceuvre de Ryle. Il faut dire d'emblée que cette étude n'est pas exhaustive. Par exemple, la célebre notion de «billet d'inférence " (inference ticket), sur laquelle je reviendrai, n'est mentionnée nulle part. D'autre part, s'il y a un sujet qui se devait d'être inclu dans un ouvrage destiné au public français et qui est malheureusement absent, c'est bien les relations entre Ryle et la phénoménologie husserlienne. L'accrochage entre Ryle et Merleau-Ponty à Royaumont est légendaire (La philosophie analytique, Paris, Éditions de Minuit, 1962), et son jugement sévère à l'endroit de la phénoménologie a profondément marqué la philosophie analytique. L'égologie transcendantale de Husserl, avec ses aspects cartésiens, n'était certes pas pour plaire à l'auteur du Concept of Mind, mais l'attitude négative de Ryle a grandement contribué à masquer pendant longtemps les liens étroits entre l'analyse frégéenne du langage et la phénoménologie husserlienne (sur ceux-ci, $c f$. M. A. E. Dummett, Les origines de la philosophie analytique, Paris, Gallimard, 1991). Ryle est d'autant plus coupable qu'il était un des rares anglais à avoir lu dès les années trente Bolzano, Brentano, Meinong, Husserl et Heidegger.

L'étude de Lucie Antoniol se divise en trois chapitres. Le premier, « Une frontière entre la signification et l'absurdité », aborde les contributions de Ryle à la philosophie de la logique et du langage. En philosophie du langage, on remarquera que Ryle s'est employé à critiquer avant tout les théories idéationnistes de Locke et Mill ( $c f$. "The Theory of Meaning », dans Collected Papers, reprint, Bristol, Thoemmes, 1990, volume II, p. 350-372). L'aspect le plus important de sa critique est le rejet du "dénotationnisme " tout azimut, qu'il développe très tôt dans "Systematically Misleading Expressions" : certaines expressions linguistiques n'ont selon lui qu' une ressemblance grammaticale avec les énoncés, leur fonction ne serait pas de référer à quelque chose (Collected Papers, op. cit., volume II, p. 56). On doit donc distinguer entre la forme grammaticale, qui est superficielle et trompeuse, et l'usage ou fonction. C'est en quelque sorte cette dernière qui détermine si l'expression est référentielle ou non.

Les diverses tentatives de contribution à la " philosophie de la logique " de Ryle furent toutes sévèrement critiquées et n'eurent peu ou pas de suites. Je prends comme exemple l'idée selon laquelle la forme logique d'une 
proposition, c'est la totalité de ses « pouvoirs logiques" (logical powers) : la signification de « si $p, q$ » se réduirait au fait qu'en conjonction avec « $p$ », on obtient " $q$ " (modus ponens) et en conjonction avec «non- $q$ ", on obtient «non- $p$ » (modus tollens). Cette définition est non seulement circulaire, elle ne s'applique pas aux conditionnels contrefactuels (J. L. Mackie, Truth, Probability and Paradox, Oxford, Oxford University Press, 1973, p. 81-82). Autre exemple, dans " "If ", "So " and "Because " " (Collected Papers, op. cit., volume II, p. 234-249), Ryle présentait une analyse particulière du conditionnel « si $p, q$ », qu'il comparait à un « passe » : un tel conditionnel nous donne la permission d'inférer " $q$ " si on a " $p$ ", tout comme un billet de train nous permet de voyager entre Oxford et Londres, à condition que nous prenions le train à Oxford. (C'est ici l'origine de l'expression «billet d'inférence ».) Cette conception a conduit Ryle à critiquer la conception traditionnelle du modus ponens, « si $p, q ; p$ donc $q$ » Le « $p$ » et le « $q$ » du conditionnel « si $p, q$ » ne peuvent qu'indiquer une place vide, ils ne sont pas assertés (il n'y a ni usage, ni mention). Le conditionnel est en quelque sorte d'une règle d'inférence " déguisée " qui ne peut pas être évaluée en termes de vérité et de fausseté. Cette conception radicale des conditionnels tombe sous le coup de la critique adressée par Peter Geach connue sous le titre de « Frege point " (cf. Logic Matters, Blackwell, Oxford, 1972, p. 254-269) : lorsque le conditionnel est inséré dans un modus ponens, l'énoncé « $p$ » doit exprimer la même pensée dans ses deux occurences, sous peine d'équivocation (même chose pour " $q$ "). On peut regarder la critique de Geach comme un point tournant de la philosophie anglaise. Elle fut suivie de la montée des théories frégéennes de la signification, qui prévalent de nos jours et qui rejetent le point de vue anti-dénotationniste de Ryle mentionné plus haut : si une expression se comporte syntaxiquement de la même manière qu'une expression référentielle, alors elle a une référence. Il n'y a pas de « règles déguisées ». Les difficultés d'un traitement vériconditionnel des conditionnels sont notoires, cependant seuls quelques logiciens « relevantistes » se sont intéressé aux idées de Ryle. A. R. Anderson et N. D. Belnap Jr. ont donné une formalisation du " ticket entailment» dans Entailment. The Logic of Relevance and Necessity (Princeton, Princeton University Press, 1975, p. 41-50).

Il ne faut pas oublier que Ryle fait partie, avec J. L. Austin et H. H. Price (surnommé affectueusement "Perception" Price), d'une génération d'oxoniens dont la formation philosophique, les « Greats », donnait une place prépondérante à la philosophie grecque et n'incluait pas de logique formelle post-frégéenne. (L'importance de cette formation se fait sentir non seulement dans les travaux de Ryle en philosophie grecque, tels que son livre Plato's Progress (Cambridge, Cambridge University Press, 1966) mais aussi dans ses travaux de logique philosophique : ses «Categories" (Collected Papers, op. cit., volume II, p. 170-184) étaient d'inspiration aristotélicienne.) Ce n'est que dans les années cinquante que la logique formelle a vraiment fait son apparition à Oxford avec la présence de Hao Wang à All Souls et de Burton Dreben, 
venu étudier avec Austin. (Le jeune Michael Dummett fut un des premiers oxoniens à l'apprendre.) En ce sens, Ryle appartient à un autre monde philosophique, loin de celui de Cambridge, dont les débats étaient hérités de l'école néo-réaliste de J. Cook Wilson et H. W. B. Joseph. Ces auteurs, Austin, Price et Ryle, étaient, tout comme leurs prédécesseurs, d'autant plus réfractaires à l'usage de la logique formelle qu'ils ne la connaissait pas. Leur contributions à l'analyse de la forme logique des énoncés en souffrit donc. La carrière de Ryle présente sur ce front l'aspect retraite rangée : de l'article de 1930 « Are there Propositions?" (Collected Papers, op. cit., volume II, p. 12-38), où Ryle confond presque absurdité et fausseté, à l'article « Use, Usage and Mention" de 1961 (Collected Papers, op. cit,, volume II, p. 407-415), Ryle a, comme le dit Antoniol, «abandonné progressivement presque toutes ses ambitions théoriques " (p. 42). Cette retraite, qui laissa le champs libre au traitement formel de la sémantique, a fortement contribué à façonner le préjugé envers la logique formelle qui marqua la " philosophie du langage ordinaire " oxonienne de l'après-guerre. Les philosophes du langage ordinaire tels qu'Austin ne s'embarassèrent point de définir les termes sémantiques (et psychologiques) qu'ils utilisaient dans leurs analyses, ce qui leur valu d'être vertement critiqués (cf. M. A. E. Dummett, «Can Analytic Philosophy be Systematic and Ought it to Be? ", dans Truth and other Enigmas, Londres, Duckworth, p. 444-445). L'étiquette de " philosophe du langage ordinaire" convient cependant aussi mal à l'œuvre de Ryle qu'à celle du second Wittgenstein et il est malheureux que le livre de Lucie Antoniol ne contribue pas à redresser ce tort.

Dans le deuxième chapitre, Antoniol présente une interprétation "sémantique » du Concept of Mind. (On retrouve déjà cette interprétation dans la recension de Margaret MacDonald, "Professor Ryle on The Concept of Mind ", dans Philosophical Review, 1951, p. 80-90.) La distinction cartésienne entre corps et esprit était ontologique. La critique de Ryle, comme Antoniol le montre bien, porte sur la sémantique du vocabulaire «mentaliste " : selon Ryle, ce vocabulaire aurait pour fonction non pas de nommer quoi que ce soit, mais de qualifier les actions et réactions, et l'erreur cartésienne consiste en l'invention d'une substance que le mot « esprit » viendrait nommer. Antoniol critique avec succès une erreur d'interprétation assez fréquente, selon laquelle Ryle serait un béhavioriste (p. 43, 62, 93-95). Pour Ryle, le béhaviorisme d'un Watson est aussi fautif que le dualisme cartésien, puisque que ce qu'un béhavioriste entend par «comportement» et «état mental » est exactement la même chose que ce qu' un dualiste cartésien entend par ces mots.

On peut reprocher à Antoniol de s'être concentrée dans ce chapitre sur les questions d'interprétation. Elle ne fait aucun cas des théories concurrantes telles que celle de Peter Geach dans Mental Acts (Londres, Routledge \& Kegan Paul, 1957), ou encore la théorie matérialiste de J. J. C. Smart ("Sensations and Brain Processes", dans C. V. Borst (dir.), The Mind-Brain 
Identity Theory, Londres, MacMillan, 1970, p. 52-66). De plus, Antoniol passe sous silence plusieurs critiques, jugées aujourd'hui fatales, du «programme » de Ryle. Par exemple, plusieurs auteurs (Chisholm, Geach et Putnam) ont montré que la notion rylienne de propriété dispositionnelle n'évite pas la circularité : un quidam peut être disposé à prendre son parapluie s'il croit qu'il va pleuvoir, mais seulement s'il désire rester sec. Ce même quidam peut être disposé à prendre son parapluie s'il désire rester sec, mais seulement s'il croit qu'il va pleuvoir. Antoniol passe aussi sous silence l'approche "fonctionaliste ", aujourd' hui orthodoxe, développée dans les années 60-70 par les Putnam, Lewis et Davidson, qui remplace l'analyse dispositionnelle par une analyse des états mentaux en terme de leurs rôles causals. (Une adaptation des énoncés de Ramsey permet d'éviter toute circularité dans les définitions ; cf. D. Lewis, "Psychophysical and Theoretical Identifications », Australasian Journal of Philosophy, vol. 50, 1972, p. 249-258.)

Depuis un quart de siècle l'influence de Ryle s'est pour ainsi dire évanouie ; sa philosophie n'offre plus qu'un intérêt historique. Toute étude portant sur Ryle doit certes nous initier à ses idées principales, mais elle doit aussi nous convaincre de leur pertinence, à défaut de vouloir se cantonner dans le domaine de l'histoire de la philosophie. Contrairement à ce que son titre annonce, l'étude d'Antoniol ne fait aucune référence aux débats contemporains en philosophie de l'esprit ; elle ne nous n'indique tout simpliment pas en quoi les idées de Ryle auraient un intérêt autre qu'historique. (Il faut dire que l'étude de William Lyons, Gilbert Ryle. An Introduction to his Philosophy (Brighton, Harvester Press, 1980) ne montre à cet égard guère plus d'intérêt.)

Le troisième chapitre du livre à pour titre "la dissolution du sujet pensant». Antoniol y présente une interprétation du concept de " personne " chez Ryle qui l'apparente à un «concept formel » au sens du Tractatus Logico-Philosophicus. Il s'agirait donc d'un concept pour lequel aucune question ontologique ne peut se poser de façon sensée. Selon moi, il s'agit ici de l'aspect le plus intéressant de l'ouvrage, mais les analyses de Lucie Antoniol auraient gagné en lucidité si elle avait évité d'établir des rapports avec les idées de Francis Jacques sur la « dimension dialogique » (p. 40-42, 72, 77). Une comparaison avec la notion de "personne " chez Strawson, présentée dans le chapitre III de Individuals (Londres, Methuen \& Co., 1959), aurait été plus appropriée. Il n'y a cependant qu'une trop brève mention de ce livre (p. 95). Antoniol décrit en tout dernier lieu l'évolution de la pensée de Ryle jusqu'à ses derniers travaux, où il présentait sa "théorie adverbiale " de la pensée. (L'exemple d'un « verbe adverbial » selon Ryle est « se dépêcher », qui présuppose une activité concrète telle que " prendre son petit-déjeûner », que ce verbe qualifie. "Penser" fonctionnerait comme un verbe adverbial.) Les quelques pages de l'étude d'Antoniol sur cette question (p. 79-88) ne divergent pas sensiblement de celles de Lyons (Gilbert Ryle.., op. cit., p. 182195). Encore une fois, les idées de Ryle, que viennent ici complèter sa théorie des « actes négatifs », font face à de sérieuses objections, laissées depuis sans 
réplique. On voit donc mal en quoi l'œuvre de Ryle n'est pas, à l'inverse de ce que pense Antoniol (p. 10), un « filon épuisé ".

Pour terminer, j'aimerais dire un mot sur la conception que Ryle se faisait de la philosophie et sur ses rapports avec Wittgenstein. L'influence du Tractatus fut déterminante pour Ryle et il n'est guère surprenant de retrouver des parallèles entre les idées développées indépendemment par ces deux philosophes dans les années trente. Les parallèles ne sont nulle part aussi flagrants que dans la lecture de "Taking Sides in Philosophy " de 1937 (Collected Papers, op. cit., volume II, p. 153-169), où Ryle présente une conception anti-cognitiviste de la philosophie presqu'en tous points similaire à celle que présentera Wittgenstein dans les Investigations philosophiques. Pour Ryle, le philosophe ne découvre pas de nouveaux faits ou de nouvelles vérités, mais son activité se résume à la production de simples truismes à propos desquels il ne peut y avoir de dispute. Le philosophe ne tente simplement que de clarifier les choses lorsque nous nous sommes empêtrés dans notre langage même. Ryle aura peut-être poussé cette idée plus à fond en développant la notion de «systematic ambiguity » dans «Systematically Misleading Expressions» (op. cit.) et dans sa conférence inaugurale "Philosophical Arguments » (Collected Papers, op. cit., volume II, p. 194-212) ; ce sont les expressions systématiquement ambiguës qui sont considérées comme sources des problèmes philosophiques. La conception anti-cognitiviste n'a aujourd'hui que peu d'adeptes, contrairement à l'approche systématique de Dummett dans "Can Analytic Philosophy be Systematic and Ought it to Be? ? (op. cit., p. 456), et il est difficile de trouver, dans les textes de Ryle comme dans l'étude d'Antoniol, de bonnes raisons d'y adhérer.

Département de philosophie

Université d'Ottawa

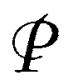

